\title{
Expanding the phenotype of metabolic cutis laxa with an additional disorder of $\mathrm{N}$-linked protein glycosylation
}

\author{
Peter Witters $\mathbb{D}^{1,2} \cdot$ Jeroen Breckpot ${ }^{3} \cdot$ François Foulquier $^{4} \cdot$ Graem Preston $^{5} \cdot$ Jaak Jaeken $^{1} \cdot$ Eva Morava ${ }^{1,2,5}$
}

Received: 18 July 2017 / Revised: 1 October 2017 / Accepted: 24 October 2017 / Published online: 30 November 2017

(c) European Society of Human Genetics 2018

Genetic syndromes associated with cutis laxa (CL) and wrinkled skin are multisystem disorders with progeroid features, including sagging, lax and wrinkled skin [1,2]. Metabolic CL is genetically heterogeneous. We previously reported on the frequently overlapping clinical phenotypes, including X-linked and autosomal recessive forms [2]. However, recently new forms of CL have been described.

"Metabolic" CL is related to different inborn errors of metabolism; the historic example is the X-linked defect in ATP7A (MIM 309400). Autosomal recessive metabolic CL types are ARCL2 and ARCL3. ARCL2 patients present with a characteristic face, skeletal and joint abnormalities, developmental and growth delay due to mutations in different genes, including ATP6VOA2 (MIM 219200, 278250), RIN2 (MIM 613075), COG7(MIM 608779), GORAB (MIM 231070), and PYCR1 (MIM 612940, 614438) [2-4]. These encode endosomal and Golgi proteins (ARCL2A), and affect trafficking and glycosylation, except for PYCRI, which is involved in mitochondrial proline synthesis (ARCL2B). ARCL3 patients have parchment-like, progeroid skin, cataracts, corneal clouding, and significant neurologic disease [5], caused by mutations in PYCRI or ALDH18A1 (MIM 616603, 219150), also involved in mitochondrial proline synthesis [2]. Additional inborn

Peter Witters

peter.witters@uzleuven.be

1 Metabolic Center, Department of Pediatrics, University Hospitals Leuven, Herestraat 49, Leuven B-3000, Belgium

2 Department of Development and Regeneration, Organ systems cluster, Faculty of Medicine, KU Leuven, Leuven B-3000, Belgium

3 Center for Human Genetics, Catholic University Leuven, Leuven B-3000, Belgium

4 Université Lille, Centre National de la Recherche Française, UMR 8576-Unité de Glycobiologie Structurale et Fonctionnelle-Unité de Glycobiologie Structurale et Fonctionnelle, Lille F-59000, France

5 Hayward Genetics Center, Tulane University School of Medicine, New Orleans, Louisiana 70112, USA errors can also be associated with CL without a clear designation to ARCL2 or ARCL3; Transaldolase deficiency (TALDO MIM 606003) and Lenz-Majewski syndrome (MIM 151050) and Cantu syndrome (MIM 239850). ATP6V0A2-CDG related CL has been described both with normal elastin histology (wrinkly skin syndrome MIM 278250) and abnormal elastin histology (ARCL2A MIM 219200). COG7-CDG is another CDG with CL and normal elastin morphology.

Recently, novel metabolic CL syndromes were added to the growing list of ARCL. Their clinical presentation highly overlaps with the phenotypic spectrum of ARCL2A $[6,7]$ and ARCL2B [8]. Mutations in ATP6V1A (MIM 617403) and ATP6VIE1 (MIM 617402), two components of the VATPase complex, were shown to cause ARCL2A, and a very similar phenotype to that seen in ATP6V0A2-CDG [7]. They have a type II pattern on transferrin isoelectric focusing (TIEF) similar to defects involving the V-ATPase complex in the Golgi, affecting glycosylation [3, 7]. In another new ARCL, lactic acidemia is the diagnostic clue for mitochondrial ECHS1 (MIM 616277) [8] deficiency.

Here we report on the previously unreported finding of CL due to a hemizygous mutation in the gene ATP6API (MIM 300972), a component of the V-ATPase complex that was recently described to cause an X-linked N-glycosylation disorder with liver disease [9].

A male patient was born at term (birth weight $3.66 \mathrm{~kg}$ (P50), length $51 \mathrm{~cm}$ (P10), and head-circumference $35 \mathrm{~cm}$ (P25)). He had CL with excess skin in the neck, axilla and groin and unilateral cryptorchidism (Fig. 1). At 2 weeks of age he had a urinary tract infection with sepsis due to Escherichia Coli. There were no underlying structural abnormalities of the urinary tract. At 2 months he developed conjugated jaundice (bilirubin direct/total 4.49/13.77 mg/d, AST $401 \mathrm{IU} / \mathrm{L}$, ALT $73 \mathrm{IU} / \mathrm{L}$, gammaGT and alkaline phosphatase were normal, ceruloplasmin was $0.03 \mathrm{mg} / \mathrm{dL}$ (normal range: $0.22-0.58 \mathrm{~g} / \mathrm{L}$ ). Stools were pigmented. Liver ultrasound showed normal bile ducts, hyperechogenic liver parenchyma, normal liver size but enlarged spleen (+3.7 SD). Urinary polyols showed an increased galactitol 


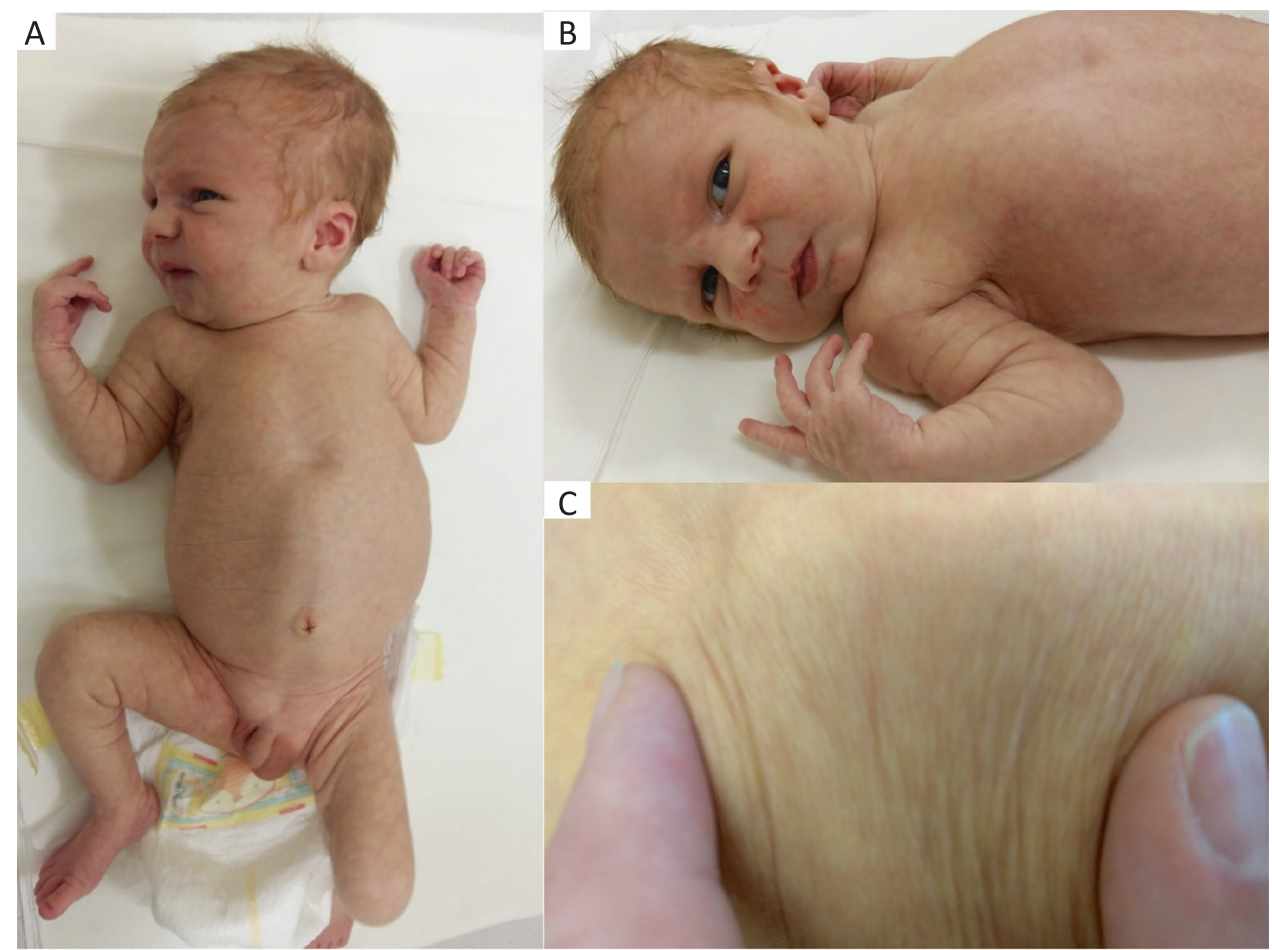

D
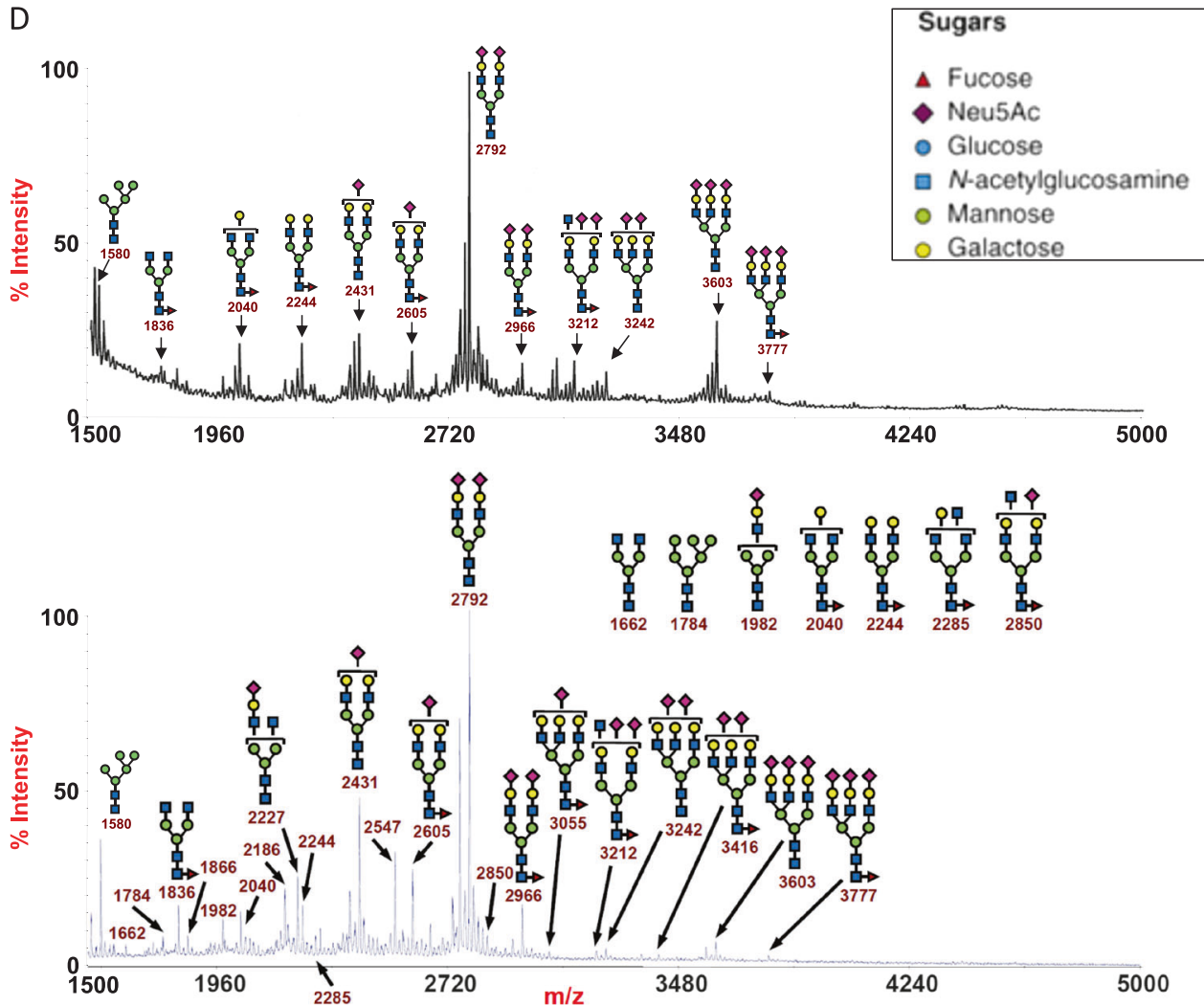

Fig. 1 a, b Presence of cutis laxa in het axillary, nuchal and inguinal region at the age of 2 weeks. There is a parchment-like appearance of the skin. c Inelastic skin appearance at the age of 2 months. Note the jaundice d MALDI-TOF-MS spectra of the permethylated N-glycans from sera of control (upper panel) and ATP6AP1-deficient individual (lower panel) carrying the homozygous c.649 T>A, mutation. MALDITOF analysis showed mainly the accumulation of undersialylated glycan suggesting a late Golgi defect. The symbols representing sugar residues are as follows: closed blue square, $\mathrm{N}$-acetylglucosamine; open green circle, mannose; closed yellow circle, galactose; open pink diamond, sialic acid ( $\mathrm{N}$-acetyl-neuraminic acid); and closed red triangle, fucose. Linkages between sugar residues have been removed for simplicity 
(227 mmol/mol creat, normal range: 3-81) but red blood cell galactose-1-P-uridilyltransferase showed a normal activity. Transaldolase deficiency was excluded.

At the age of 2 months there was a normal psychomotor development with increasing hepatosplenomegaly and improvement of lax skin. TIEF showed a type 2 pattern. A liver biopsy showed micronodular cirrhosis with a ductular reaction and steatosis. There were PAS-positive globules within the hepatocytes.

At the age of 5 months he presented with pneumonia. Chest X-ray showed an anterior diaphragmatic hernia (Morgagni). This was surgically corrected. A skin biopsy performed during surgery showed a normal amount and texture of elastin fibers.

Cardiac ultrasound showed a dilatation of the sinus aortae (18 mm, Z-score 2.85).

MALDI-TOF analysis showed truncated glycans with mainly desialylation suggesting a Golgi trafficking defect. Sanger sequencing of the ATP6AP1 gene (MIM 300197, Xq28) revealed a variant: c.649 T>A, p.(Tyr217Asn) (NM_001183.4). This is a highly conserved locus down to zebrafish. This maternally inherited variant was predicted to be pathogenic (0.999 Polyphen-2 and SWIFT) and has not been reported in normal population databases (local, ExAc, and GnomAD), nor in other patients with CDG. The data from this study were submitted to the LOVD database (http://www.lovd.nl/3.0/home) (individual \#00131880).

The V-ATPase complex is essential for the Golgiendosomal secretory pathway, and for the normal maturation of extracellular matrix components [3, 7, 9]. Components of the V-ATPase complex, causing CL when deficient, are summarized in Table 1.

Interestingly, several components and assembly factors of this same V-type ATPase have been recently described in CDGs that primarily affect the liver. TMEM199-CDG (MIM 616829) and CCDC115-CDG (MIM 616828) are Vtype ATPase assembly factors that localize to the endoplasmic reticulum [10]. TMEM199-CDG is characterized by an adult-onset mild liver disease consisting of liver steatosis and subclinical transaminase elevations [10]. In CCDC115-CDG there are increased transaminases, evolving to liver cirrhosis and liver failure. Developmental delay, hypotonia and seizures are described [10]. ATP6AP1 and ATP6AP2 are both accessory subunits of the VATPase. Their phenotypes are largely overlapping. However, mutations in ATP6AP2 (MIM 300556), a regulatory subunit of the V-ATPase super-complex, were recently shown to present as metabolic CL [11]. The presence of CL in ATP6AP1-CDG has not been reported previously, although most patients described were older than 1 year of age an might have outgrown skin wrinkling (only one patient showed localized nuchal wrinkled skin in adulthood; personal communication) [9].

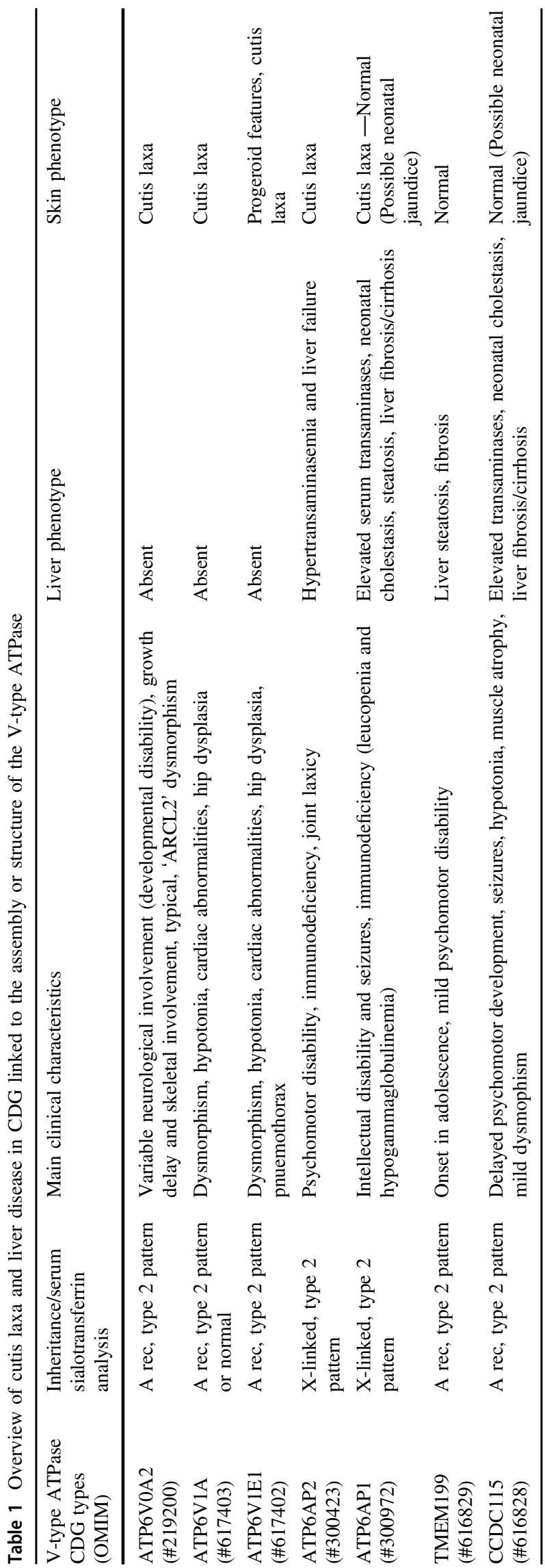


ATP6AP1-CDG presents with obligate liver disease (ranging from increased transaminases to cholestasis and liver cirrhosis) in association with neurological disease in $60 \%$ (epilepsy, developmental disability) and immunodeficiency (frequent infections, leukopenia and low immunoglobulin levels) [9]. Although biochemical parameters for immunodeficiency were normal in our patient, he did have a urinary tract sepsis, pneumonia and several viral infections often with a relatively high C-related peptide. Interestingly, after the cholestasis disappeared, the biochemical signs of ATP6AP1-CDG (low ceruloplasmin and elevated cholesterol) normalized.

The combination of hepatopathy and hypercholesterolemia with immune abnormalities is a recognizable phenotype, which should initiate TIEF analysis. The combination of these features with CL in our patient lead to direct Sanger sequencing and the genetic diagnosis.

In the current report, we extend the phenotypic spectrum of ATP6AP1-CDG with connective tissue manifestations (CL, dilation of the aortic root, diaphragmatic hernia, and wrinkled skin) and suggest that ATP6AP1-CDG should be considered a possible cause of X-linked CL.

Funding PW and JB are supported by the clinical research fund, University Hospitals Leuven, Leuven, Belgium.

\section{Compliance with ethical standards}

Conflict of interest The authors declare that they have no competing interests.

\section{References}

1. Morava E, Guillard M, Lefeber DJ, Wevers RA. Autosomal recessive cutis laxa syndrome revisited. Eur J Hum Genet. 2009;17:1099-110.

2. Gardeitchik T, Mohamed M, Fischer B, et al. Clinical and biochemical features guiding the diagnostics in neurometabolic cutis laxa. Eur J Hum Genet. 2014;22:888-95.

3. Kornak U, Reynders E, Dimopoulou A, et al. Impaired glycosylation and cutis laxa caused by mutations in the vesicular $\mathrm{H}$ +-ATPase subunit ATP6V0A2. Nat Genet. 2014;40:32-4.

4. Dimopoulou A, Fischer B, Gardeitchik T, et al. Genotypephenotype spectrum of PYCR1-related autosomal recessive cutis laxa. Mol Genet Metab. 2013;110:352-61.

5. Wolthuis DF, van Asbeck E, Mohamed M, et al. Cutis laxa, fat pads and retinopathy due to ALDH18A1 mutation and review of the literature. Eur J Paediatr Neurol. 2014;18:511-5.

6. Van Asbeck E, Wolthuis DF, Mohamed M, et al. A novel phenotype associated with cutis laxa, abnormal fat distribution, cardiomyopathy and cataract. Am J Med Genet A. 2014;164A:1049-55.

7. Van Damme T, Gardeitchik T, Mohamed M, et al. Mutations in ATP6V1E1 or ATP6V1A Cause Autosomal-Recessive Cutis Laxa. Am J Hum Genet. 2017;100:216-27.

8. Balasubramaniam S, Riley LG, Bratkovic D, et al. Unique presentation of cutis laxa with Leigh-like syndrome due to ECHS1 deficiency. J Inherit Metab Dis. 2017;14-16. https://doi.org/10. 1007/s10545-017-0036-4.

9. Jansen EJ, Timal S, Ryan M, et al. ATP6AP1 deficiency causes an immunodeficiency with hepatopathy, cognitive impairment and abnormal protein glycosylation. Nat Commun. 2016;7:11600.

10. Marques-da-Silva D, Dos Reis Ferreira V, Monticelli M, et al. Liver involvement in congenital disorders of glycosylation (CDG). A systematic review of the literature. J Inherit Metab Dis. 2017;40:195-207.

11. Cannata Serio M. Mutations in ATP6AP2 are associated to congenital disorders of glycosylation with autophagic defects. Proceedings ISCDGS2017. https://gbiomed.kuleuven.be. 2017. 